\title{
Reliability of impression cytology for the diagnosis of ocular surface squamous neoplasia employing the Biopore membrane
}

\author{
Derek M Tole, Penelope A McKelvie, Mark Daniell
}

\begin{abstract}
Aim-To evaluate the accuracy of impression cytology employing a Biopore membrane device in the diagnosis of ocular surface squamous neoplasia (OSSN).

Methods-The histology of patients undergoing excision biopsy for the suspected diagnosis of OSSN over a 20 month period was compared with the reported cytology of impression cytology specimens obtained preoperatively using the Biopore
\end{abstract} membrane device.

Results-25 excision biopsies were performed for suspected OSSN. There was accurate correlation in 20 out of the 25 cases $(80 \%)$. In three cases $(12 \%)$, there was poor correlation in that only a few dysplastic cells and hyperkeratosis were noted on cytology, whereas histology showed keratinising dysplasia in two cases and a moderately differentiated keratinising squamous cell carcinoma in the third. There were two cases $(8 \%)$ of noncorrelation-one keratinising dysplasia on histology, but anucleate keratin, squamous metaplasia, and rare atypical cells on cytology, and another case with very mild focal dysplasia on histology but squamous metaplasia on cytology. Review of the cytology showed that dysplastic cells were either rare or absent from the cytology specimens in these two cases; it was felt that these represented sampling problems rather than true false negatives. There were no false positives on cytology. Final diagnosis on histology was squamous cell carcinoma in one; carcinoma in situ in two; keratinising dysplasia in 15, non-keratinising dysplasia in seven. Conclusion-Impression cytology employing the Biopore membrane accurately predicts the histological diagnosis of OSSN. The presence of hyperkeratosis, inflammatory cells with only a few dysplastic cells, may indicate high grade keratinising dysplasia or squamous cell carcinoma. The method is both rapid and easy to perform in routine clinical practice. These findings suggest that there may be a role for its use in the initial assessment and follow up of patients with suspected OSSN.

(Br f Ophthalmol 2001;85:154-158)

Egbert et al first introduced ocular surface impression cytology into ophthalmology in 1977. ${ }^{1}$ These authors used cellulose acetate fil- ter paper for the collection of the superficial layer of cells from conjunctiva. This method has subsequently been modified by several authors $\mathrm{s}^{2-7}$ for investigation of dry eyes, staging conjunctival squamous metaplasia, diagnosis of vitamin A deficiency, ocular cicatricial pemphigoid, limbal stem cell failure, microbiological and viral infections, and the diagnosis of ocular surface squamous neoplasia. Nolan et al first reported on the application of impression cytology using acetate paper for the diagnosis of conjunctival neoplasms. ${ }^{7}$ However, until 1997, most studies used multiple specially cut strips which were placed in special multiwell sample containers to be transported to the laboratory, a procedure which most ophthalmologists found rather cumbersome, too time consuming and therefore unsuitable for use in routine outpatient clinics. ${ }^{8}$ More recently, Thiel et al have described the use of a new Biopore membrane device (Millicell-CM 0.4 $\mu \mathrm{m}$ PICM 012550, Millipore Corp, Bedford, MA, USA) for obtaining conjunctival cells for the diagnosis of superficial viral infections. ${ }^{6}$ It offers the advantage of being quicker and easy to use in routine clinical practice. The device is mechanically stable allowing direct orthogonal sampling from the ocular surface. There is good adhesion of cells to the Biopore membrane so that a large layer of cells is easily harvested. The membrane itself is transparent in the wet state which allows for a detailed cytological examination. We report its role in the diagnosis of OSSN.

\section{Methods}

Impression cytology specimens using the Biopore membrane were obtained preoperatively from all patients undergoing a formal excisional biopsy for suspected OSSN over a 20 month period from May 1998 to January 2000.

The Biopore membrane disc $(8 \mathrm{~mm}$ in diameter) is attached to a small plastic tube

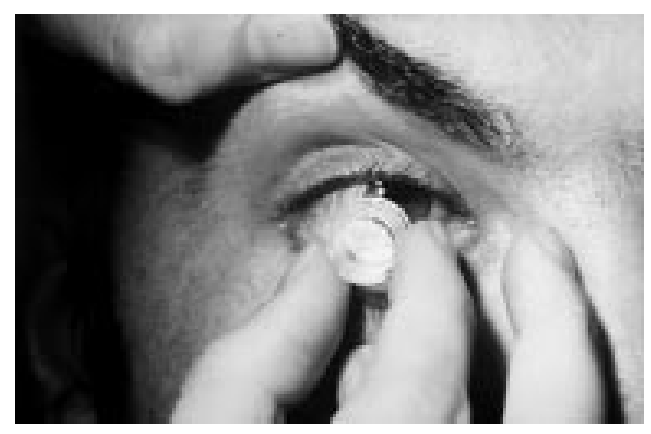

Figure 1 Impression cytology sampling using the Biopore membrane device. 


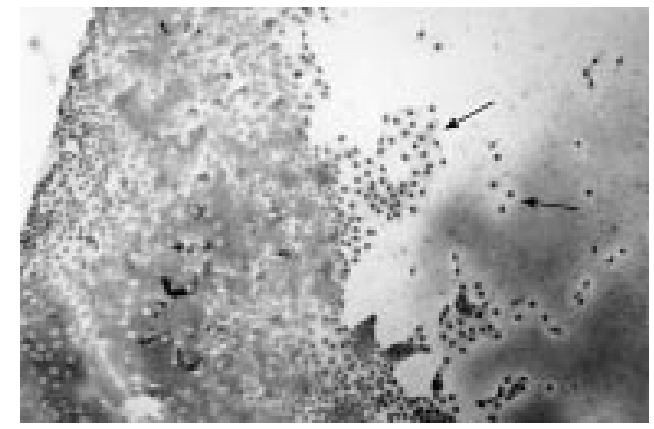

Figure 2 Normal ocular surface impression cytology including the limbus. To the left, there are sheets of conjunctival epithelial cells with goblet cells (clear spaces) which merge with the limbus centrally and on the right, corneal epithelial cells which are larger (arrows). Giemsa. Magnification $\times 200$.

and comes wrapped in a sterile package. Before use, three protruding plastic legs must be snapped off from the base of the tube with a pair of Spencer-Wells forceps. Oxybuprocaine eye drops $0.4 \%$ are then instilled into the lower fornix. Cytology specimens are obtained from the conjunctiva or cornea. The membrane device is firmly pressed against the area to be sampled until the membrane becomes translucent (Fig 1). This takes on average 10-20 seconds. The device is then immediately transferred into a $20 \mathrm{ml}$ container of $95 \%$ alcohol without air drying, taking care to fully immerse the membrane. On reaching the laboratory, the specimen is stained with haematoxylin and eosin, then cut from the tube and mounted on a slide ready for interpretation. The Biopore membrane at this stage is fully transparent and allows a detailed cytological examination (Fig 2 , normal ocular surface impression cytology). In the early period of this series, the filters were air dried and stained with Giemsa. However, because of rapid fading of the stain, it was decided to fix the filters and use a haematoxylin and eosin stain. A single observer (PMcK) interpreted all cytology specimens without previous knowledge of the results of the excision biopsy reports. The cytology was reported for dysplasia, where cells with enlarged hyperchromatic nuclei with increased nuclear/cytoplasmic ratio, irregular nuclear contour, and coarse nuclear chromatin + prominent nucleoli (Figs 3 and 4), squamous metaplasia of conjunctiva (abundant cytoplasm, reduced nuclear/cytoplasmic ratio, pyknotic nuclei) and hyperkeratosis (anucleate keratin (Fig 5), parakeratotic debris, dyskeratotic cells and keratohyaline granules) were seen. An attempt to grade the dysplasia on cytology was not made, but numbers of dysplastic cells were noted in semiquantitative terms (occasional, few, moderate, numerous). Although the surgical biopsies were initially reported by different pathologists, all biopsies were reviewed by $\mathrm{PMcK}$, blinded to cytology results, for this study. A comparison of the findings for both the cytology and histological specimens was made addressing in particular the accuracy of impression cytology in predicting the presence of dysplasia, carcinoma in situ, and invasive squamous cell carcinoma.

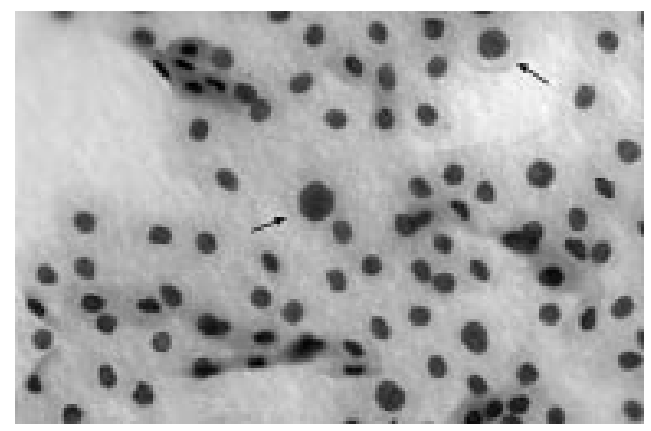

Figure 3 Occasional dysplastic cells (arrows) in a case of keratinising dysplasia (case 2). Giemsa. Magnification $\times 2000$

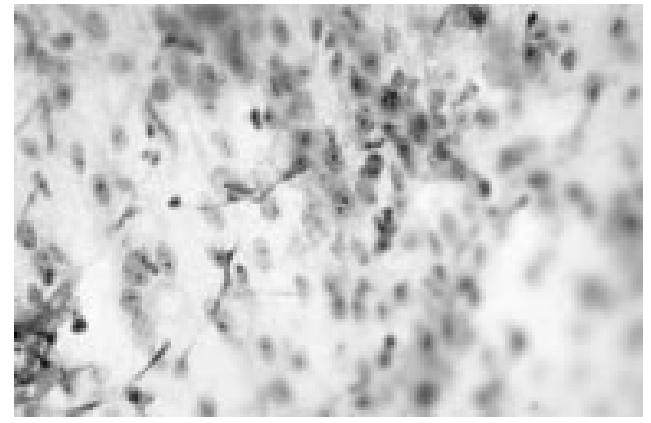

Figure 4 Numerous dysplastic cells with prominent nucleoli in severe non-keratinising dysplasia (case 23). Haematoxylin and eosin. Magnification $\times 2000$.

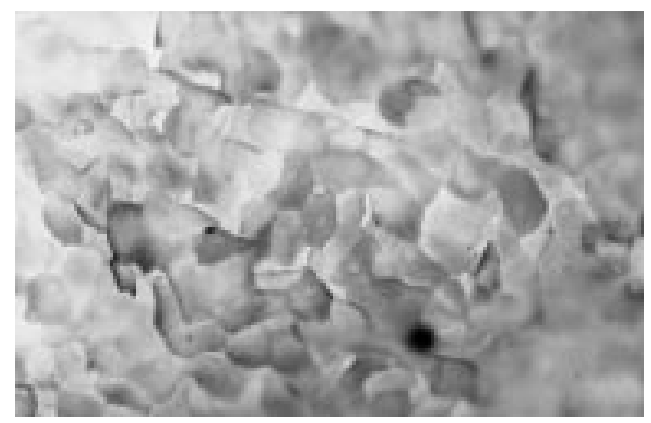

Figure 5 Anucleate keratotic debris on impression cytology in a case of keratinising dysplasia (case 2). Giemsa. Magnification $\times 2000$.

\section{Results}

A total of 25 excision biopsies in 24 patients were performed for suspected OSSN with results listed in Table 1. One patient (JA) had two episodes separated by 9 months of suspected and biopsy proved OSSN. There were 19 males, aged between 31 and 81 with a median of 68 years, and five females aged between 41 and 83 years (median 60 years). The median age for the whole group was 68 years. Patient age according to decade included: 30-39 (one); 40-49 (three); 50-59 (two); 60-69 (eight); 70-79 (seven); 80-89 (three). Twenty eight impression cytology specimens were obtained preoperatively by 15 different ophthalmologists or ophthalmologists in training over the course of the series. Three patients had two separate preoperative impression cytology samples. The reasons for this were that in one patient (JB) the first specimen was suboptimal with very few cells, but the second sample was positive for dysplasia. In a second patient $(\mathrm{HF})$, the first specimen was negative 
Table 1 Cytological and histopathological reports for comparison

\begin{tabular}{|c|c|c|c|}
\hline Number & Cytology & Histology & Comments \\
\hline 1 GE $M, 74$ & Dysplasia & Mild to moderate dysplasia & Correlation \\
\hline $2 \mathrm{RD} M, 31$ & Keratin + few dysplastic cells & Severe keratinising dysplasia & Poor correlation \\
\hline 3 GF M, 72 & $\begin{array}{l}\text { Few dysplastic cells }+ \\
\text { inflammatory cells }\end{array}$ & $\begin{array}{l}\text { Moderately differentiated keratinising invasive } \\
\text { squamous cell carcinoma }\end{array}$ & Poor correlation \\
\hline 4 RC M, 80 & Dysplasia & Mild dysplasia & Correlation \\
\hline 5 FMcC M, 67 & Dysplasia & Mild dysplasia & Correlation \\
\hline 6 DT F, 73 & Dysplasia & Mild dysplasia & Correlation \\
\hline 7 RP M, 67 & Dysplasia & Mild dysplasia & Correlation \\
\hline $8 \mathrm{MR}$ F, 54 & Dysplasia & Moderate dysplasia & Correlation \\
\hline $9 \mathrm{CS} \mathrm{F}, 83$ & Keratinising dysplasia & Moderate to severe keratinising dysplasia & Correlation \\
\hline $10 \mathrm{JB}$ M, 69 & Keratinising dysplasia & Severe keratinising dysplasia & Correlation \\
\hline 11 BR M, 66 & Keratinising severe dysplasia & Severe keratinising dysplasia & Correlation \\
\hline 12 PC M, 49 & Keratinising dysplasia & Severe keratinising dysplasia & Correlation \\
\hline 13 JA $M, 40$ & Keratinising dysplasia & $\begin{array}{l}\text { Moderate/severe dysplasia with focal } \\
\text { parakeratosis }\end{array}$ & Correlation \\
\hline 14 EV M, 61 & Keratinising severe dysplasia & Severe keratinising dysplasia & Correlation \\
\hline 15 AV F, 60 & Keratinising dysplasia & Moderate keratinising dysplasia & Correlation \\
\hline 16 GP M, 50 & Keratinising dysplasia & Moderate to severe keratinising dysplasia & Correlation \\
\hline 17 MV M, 74 & $\begin{array}{l}\text { Keratin, squamous metaplasia, } \\
\text { occasional atypical cells. }\end{array}$ & Moderate to severe keratinising dysplasia & NON correlation \\
\hline $18 \mathrm{KL} \mathrm{M}, 74$ & Squamous metaplasia & Very mild focal dysplasia & 18. NON correlation \\
\hline 19 JT M, 72 & $\begin{array}{l}\text { Hyperkeratosis with few } \\
\text { dysplastic cells. Rider re KD }\end{array}$ & Severe keratinising dysplasia/ carcinoma in situ & Possible correlation \\
\hline $20 \mathrm{HF}$ M, 81 & $\begin{array}{l}\text { Keratin and a small number of } \\
\text { dysplastic cells }\end{array}$ & Mild to focally moderate keratinising dysplasia & Correlation \\
\hline 21 PA F, 41 & Keratinising dysplasia & Keratinising severe dysplasia & Correlation \\
\hline 22 CB M, 63 & Keratinising dysplasia & Keratinising severe dysplasia & Correlation \\
\hline 23 EB M, 68 & High grade dysplasia & Severe dysplasia wth focal carcinoma in situ & Correlation. \\
\hline 24 JA M, 40 & Keratinising dysplasia & Keratinising severe dysplasia & Correlation \\
\hline 25 GT M, 79 & High grade keratinising dysplasia & Keratinising severe dysplasia & Correlation \\
\hline
\end{tabular}

for dysplasia (assumed to be sampling error), but the second sample was positive for dysplasia. The third patient (AV) had two impressions positive for dysplasia. Biopsies confirmed the cytological diagnosis in 20 cases $(80 \%)$. In three cases $(12 \%)$, there was poor correlation in that only few dysplastic cells and hyperkeratosis were noted on cytology, whereas histology showed keratinising dysplasia in two cases and a moderately differentiated keratinising squamous cell carcinoma in the third. There were two cases $(8 \%)$ of non-correlation-one keratinising dysplasia on histology (Fig 6) but anucleate keratin, squamous metaplasia, and only rare atypical cells on cytology (Figs 3 and 5 ), and another case with very mild focal dysplasia on histology but squamous metaplasia on cytology. Review of the cytology showed that dysplastic cells were either rare or absent from the cytology specimens, and that these represented sampling problems rather than true false negatives. There were no false positives on cytology. Final diagnosis on histology was squamous cell carcinoma in one, carcinoma in situ in two, keratinising dysplasia in 15, non-keratinising dysplasia in seven (Fig 7). In cases of dysplasia, a grade was not given on the cytology specimen but numbers of dysplastic cells were indicated in semiquantitative terms. Cases of low grade dysplasia had few abnormal cells, whereas severe non-keratinising dysplasia often showed sheets of abnormal cells. Over the period of the study, the pathologist obtained expertise with the cytological findings in keratinising dysplasias. In the latter part of the study if only a small number of dysplastic cells were obtained in addition to evidence of hyperkeratosis (anucleate squames, keratohyaline granules, dyskeratotic cells), a rider was added to the final opinion that keratinising dysplasias may only yield few abnormal cells on impression cytology compared with the nonkeratinising dysplasias.

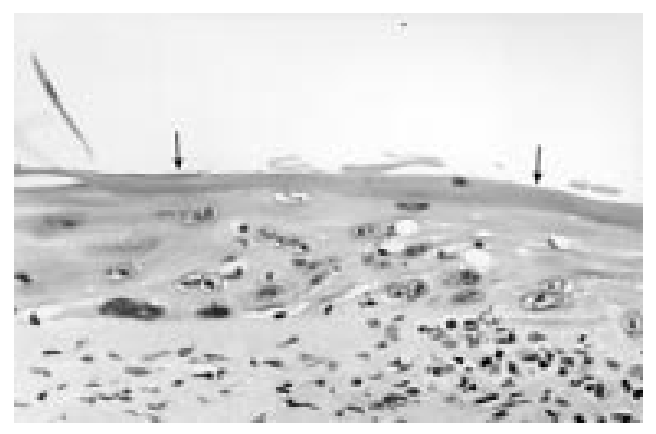

Figure 6 Histology of keratinising dysplasia showing thick surface layer of hyperkeratosis, indicated by arrows (case 2). Haematoxylin and eosin $\times 2000$.

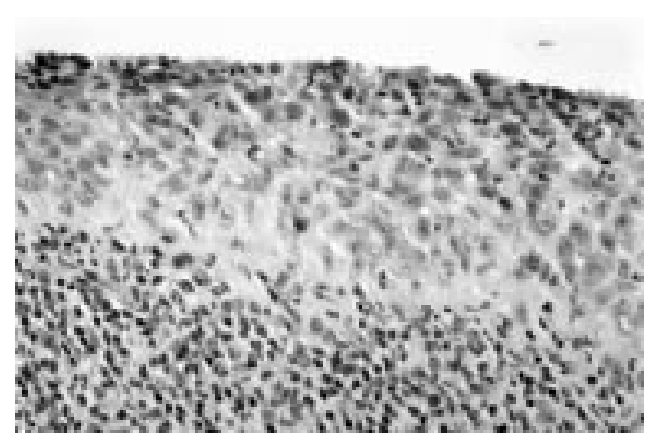

Figure 7 Histology of severe non-keratinising dysplasia with dense stromal inflammatory infiltrate and inflammation in the epithelium (case 23). Haematoxylin and eosin $\times 2000$.

\section{Discussion}

Impression cytology employing the Biopore membrane accurately predicts the histological diagnosis of ocular surface squamous neoplasia (OSSN) with a high correlation rate of $80 \%$ (20/25) for predicting the subsequent histological findings. Nolan et al had a similarly high predictability rate of $77 \%(55 / 71)$ for their group of moderate dysplasia to microinvasive carcinoma in their series of patients using cellulose acetate filter paper. $^{7}$ These authors 
commented that impression cytology was less sensitive for cases of invasive squamous cell carcinoma. However, they noted as in our series, the presence of keratin, inflammatory cells, and the paucity of diagnostic cells in such cases, thus alerting the clinician to the possibility of disease at the severe end of the spectrum. The presence of abundant surface keratin in either a keratinising dysplasia or squamous cell carcinoma may prevent an accurate sampling of representative dysplastic cells. For example, case 2 occurred early in the series when it was not appreciated by the pathologist that keratinising dysplasia might only yield few atypical cells. Therefore, the inexact correlation in the cases of keratinising dysplasias occurred early in the series when the pathologist was relatively inexperienced with regard to the interpretation of ocular surface cytology.

Before the introduction of Biopore membranes for ocular surface impression cytology, the use of cellulose acetate strips with special multiwell containers for transport to the laboratory was time consuming and often required a dedicated person to perform the procedure and a series of steps in the laboratory to produce the final cleared stained filter for interpretation. This has deterred many ophthalmologists and pathology laboratories from employing this technique. ${ }^{8}$ By comparison, the new Biopore membrane device is easy and rapid to use in routine clinical practice. In our study, 15 different ophthalmologists or ophthalmologists in training performed the technique with adequate specimens obtained in over $96 \%(27 / 28)$ of cases. Special containers for transport to the laboratory are not required and the "kit" containing sterile filter, instructions, and routine $20 \mathrm{ml}$ specimen jar filled with $95 \%$ alcohol can be sent out to ophthalmologists in more remote centres and posted back to the laboratory for cytological assessment. Disadvantages include the size of the filter which may not provide such accurate four quadrant sampling as the smaller strips.?

One of the difficulties for the pathologist interpreting the specimens lies in the paucity of literature regarding criteria for diagnosis of ocular surface squamous neoplasia by ocular surface impression cytology, as highlighted by Nolan et al. ${ }^{9}$ These authors emphasise that the cytology of normal ocular surface epithelium does not show strong resemblance to that of the normal uterine cervix. Differences include the limbic epithelium, the transition zone between cornea and conjunctiva, from where corneal stem cells are derived and where most OSSN arises. At the limbus the sheets of stratified cuboidal epithelium with goblet cells of conjunctiva merge with the larger squamous corneal cells, which are usually shed singly or in small clusters (Fig 2). However, the universal cytological criteria for dysplasia-nuclear enlargement, hyperchromasia, irregular nuclear outline, coarse nuclear chromatin, and prominent nucleoli- can be applied to diagnosis of lesions of the ocular surface. Squamous metaplasia + dysplastic epithelial changes is common in sun damaged conjunctiva and limbus and has to be distinguished from normal corneal epithelium. Similarly, keratinising lesions are common $(68 \%)$ in the spectrum of OSSN seen by us compared with a much lower incidence of keratinising dysplasias in the uterine cervix. As illustrated by our series, keratinising dysplasias and squamous cell carcinomas of the ocular surface may yield very few or rare atypical cells on impression cytology. In these cases, where there is hyperkeratosis, but few dysplastic cells, adding a rider to the final opinion that only very small numbers of dysplastic cells may be found in cytological specimens of keratinising dysplasias and squamous cell carcinomas should provide useful information to the referring ophthalmologist. Thus, where impression cytology of a suspicious hyperkeratotic lesion is negative, diagnosis should be confirmed by surgical biopsy.

Another area of difficulty in impression cytology is that of differentiating in situ carcinoma from infiltrating carcinoma, since squamous cell carcinomas have been found to represent up to $21 \%$ of biopsy proved OSSN seen in Australia. ${ }^{10}$ Although the presence of prominent nucleoli has been suggested to indicate invasive carcinoma, ${ }^{7}$ we have found that to be not always reliable, since we have seen prominent nucleoli in a recent case of CIS/ severe dysplasia, where we could not demonstrate invasion in multiple serial sections (Figs 3 and 7). However, our cytological experience with squamous cell carcinoma is limited as our series only included one case.

Our findings suggest that there may be a role for the use of the Biopore membrane device in the assessment and follow up of patients with suspected OSSN. One of the main limitations to the technique is the expertise of the pathologist in assessment of the unique cytology of the ocular surface. It is optimal for the pathologist or cytopathologist to have a familiarity with normal histology and histopathology of the ocular surface. Expertise in impression cytology is acquired by continuing experience including close review, correlation with all subsequent histology specimens, and clinicopathological correlation. This enables the cytopathologist to gain familiarity and become cognizant of the difficult areas such as keratinising lesions.

Our studies have also been aided by simultaneous provision of clinical photographs such as Polaroids or digital photographs at the time of cytology sampling by two of the referring ophthalmologists.

One of the reasons for developing the technique of impression cytology is that it is a simple and practical non-invasive technique with a high level of sensitivity for diagnosis of OSSN. OSSN is a disease affecting predominantly the limbus in elderly individuals and squamous cell carcinoma of the ocular surface is a low grade malignancy. These elderly patients may not always be medically fit for biopsy, or as in the authors' experience, may refuse biopsy for a disease that is not going to reduce their life expectancy. It is recognised 
that OSSN not only occurs in multiple sites (field change effect), but has a tendency for recurrence. Since the limbal stem cells, which are responsible for renewal of the corneal epithelium throughout life, are potentially reduced in number with each surgical biopsy in these patients, impression cytology offers a safer alternative to diagnosis than repeated biopsies. In cases of recurrent dysplasia where mitomycin $\mathrm{C}$ therapy is being contemplated, a biopsy would also delay institution of therapy, as the site would have to heal before the drops could safely be given. The Biopore membrane device may also be used in the confirmation of diagnosis, when excisional biopsy is not required such as follow up in those patients who have been treated with alternative treatment methods to excision biopsy, such as mitomycin. ${ }^{11}$ However, it must be noted that cytological changes mimicking malignancy (nuclear enlargement and nuclear hyperchromasia/smudging of chromatin) have been recently reported in conjunctival biopsies up to 6 weeks following topical mitomycin C therapy for primary acquired melanosis. ${ }^{12}$ Nevertheless, there are features which can help to differentiate these changes from true dysplasia. Similar to changes seen in urothelium after topical mitomycin, the affected conjunctival epithelial cells show proportionate increase in both cytoplasm and nucleus, preserving the normal nuclear/cytoplasmic ratio, unlike the situation of increased N/C ratio in dysplasia or malignancy. Furthermore, the nuclei in mitomycin affected cells in conjunctiva and urothelium are described as showing smudgy chromatin rather than the frank hyperchromasia associated with dysplastic cells. In our experience of the impression cytology specimens following mitomycin treatment of cervical intraepithelial neoplasia (CIN), occasional atypical cells with disintegrating and apoptotic nuclei have been noted several weeks after the end of treatment, but in cases with recurrent CIN, sheets of dysplastic cells were present. Only small numbers of superficial cells were affected in the conjunctival biopsies at 6 weeks after mitomycin therapy illustrated by Salomão and colleagues, ${ }^{12}$ correlating with our experience of occasional cells on impression cytology, compared with the much larger numbers including sheets in a high grade dysplastic process. Although mitomycin related changes that have been reported persist in urothelium for years after chemotherapy, it would be interesting to study whether a similar prolonged effect occurs in the epithelium of the ocular surface.

In conclusion, we have presented data demonstrating the utility and practicality of impression cytology using the Biopore membrane device in the initial diagnosis and follow up of ocular surface squamous neoplasia. A high degree of accuracy may be obtained once the pathologist has gained expertise with the specialised cytology of the ocular surface and is aware of the limitations of this technique in dysplastic lesions with hyperkeratosis.

The authors would like to thank Mrs Usha Rane and Ms Kerryn Jones of the cytology department, St Vincent's Hospital for their help in preparation of the biopore membranes, particularly in the early stages where some troubleshooting was required. Dr McKelvie would like to thank Glenda Nolan for her generous assistance and advice regarding the technique of impression cytology and for helpful discussions. Thanks expecially to Dr Nibaran Gangopadhyay whose early contribution in collecting cases was invaluable. Thanks also to Joss Dimock of medical photography at the Royal Victorian Eye and Ear Hospital for help in preparing the illustrations.

1 Egbert PR, Lauber S, Maurice DM. A simple conjunctival biopsy. Am f Ophthalmol 1977;84:798-801.

2 Nelson DJ, Havener VR, Cameron JD. Cellulose acetate impressions of the ocular surface: dry eye states. Arch Ophthalmol 1983;101:1869-72.

3 Tseng SCG. Staging of conjunctival metaplasia by impression cytology. Ophthalmology 1985;92:728-33.

4 Natadisastra G, Wittpenn JR, West KP, et al. Impression Natadisastra G, Wittpenn JR, West KP, et al. Impression
cytology for detection of vitamin A deficiency. Arch cytology for detection of vi
Ophthalmol 1987;105:1224-8.

5 Puangsricharern V, Tseng SC. Cytological evidence of corneal diseases with limbal stem cell deficiency. Ophthalmology 1995;102:1476-85.

6 Thiel MA, Bossart W, Bernauer W. Improved impression cytology techniques for the immunopathological diagnosis of superficial viral infections. $\mathrm{Br} \mathcal{F}$ Ophthalmol 1997;81: 984-8.

7 Nolan GR, Hirst LW, Wright RG, et al. Application of impression cytology to the diagnosis of conjunctival neoplasms. Diagnostic Cytopathology1994;11:246-9.

8 Dart J. Impression cytology of the ocular surface-research tool or routine clinical investigation? (Editorial.) $\mathrm{Br} F \mathrm{Oph}$ thalmol 1997;81:989.

9 Nolan GR, Hirst LW, Bancroft BJ. Efficacy of a training programme designed to teach cervical smear screeners to identify ocular surface squamous neoplasia using conjunctival impression cytology. Cytopathology 1997;8:388-96.

10 Lee GA, Hirst LW. Retrospective study of ocular surface neoplasia. Aust NZ F Ophthalmol 1997;25:269-76

11 Frucht-Pery J, Sugar J, Baum J, et al. Mitomycin C treatment for conjunctival-corneal intraepithelial neoplasia. A multi-centre experience. Ophthalmology 1997;104: 2085-93.

12 Salomão DR, Mathers WD, Sutphin JE, et al. Cytologic changes in the conjunctiva mimicking malignancy after topical mitomycin C chemotherapy. Ophthalmology 1999; 106:1756-61.1 